\title{
11 Submitting a paper
}

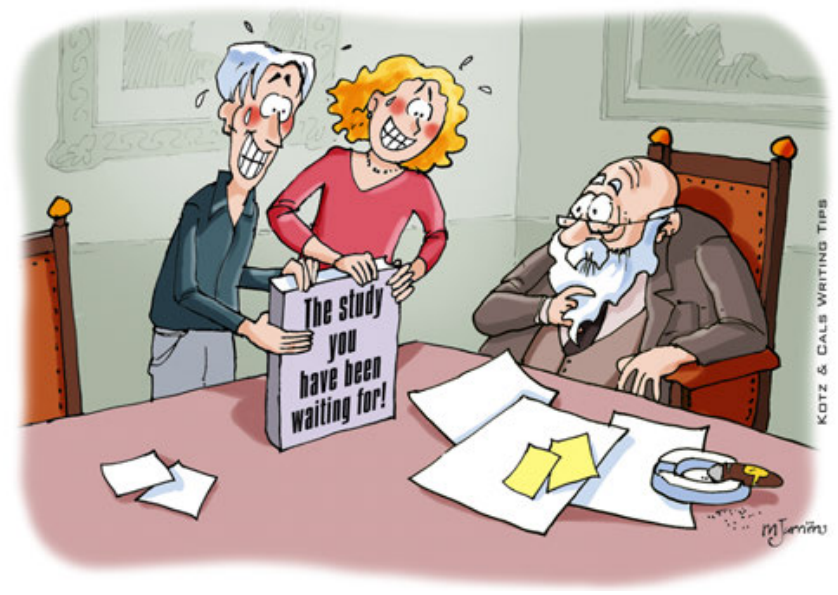

Tip 11 - Submitting a paper: write a convincing cover letter!

\section{What you should know}

There are uniform requirements for papers submitted to biomedical journals (www.icmje.org). In addition, each journal has its own specific requirements for papers, which can be found in the author instructions on the journal's website. Every journal has slightly different requirements regarding aspects like the maximum number of words, section headings and subheadings, and the reference style. Some journals ask for additional information such as a section on what is already known on the topic and what this study adds. It is advantageous to be aware of such requirements at an early stage of writing, as you want your co-authors to read, comment on, and accept these additional features as well.

The submission of your paper is usually accompanied by a cover letter. It should stress the significance of the paper for the field of research and its relevance to the specific journal. The cover letter also confirms your adherence to the journal's author requirements and contains any additional information which may be of interest to the editor such as disclosure of prior releases of data and related work. 
A journal's online submission system will guide you through the submission process step-by-step. This can be very time-consuming unless you prepare yourself well by studying all the steps of the system at an earlier stage and preparing text elements and files exactly as required by the specific journal. Most submission systems show your paper's current status in the journal's process. A good journal will make a decision about a straight rejection of your paper (i. e., an editorial decision without peer review) within 1-3 weeks after submission, or will make a decision based on reviewer comments ("reject with review" or "revise and resubmit") within 2-3 months.

\section{What you should do}

Ask yourself if you are fully satisfied with the manuscript. Have you taken sufficient time for reflection since you finished the last bits? Read your entire manuscript carefully one last time, preferably after letting it rest for a couple of days. This enables you to take a fresh look at your own work. When reading your manuscript for the last time, ask yourself: is the storyline obvious, logical and interesting? Is the text clear but also concise? Have I been consistent in the use of terms? Is the language correct and are there zero typos left?

Consider having your manuscript proof-read by a trusted peer. This is a researcher or a non-scientist with editorial experience whom you know well, who has the basic scientific knowledge that you would assume from the readers of the journal you are about to submit your manuscript to, but who has not been involved in your study or the writing of the manuscript. Such a peer is able to identify the "blind spots" in the manuscript which you and your co-authors may have overlooked, and to give valuable feedback for final improvements to the manuscript. Furthermore, if your mother tongue is not English, it can be useful to have your paper checked by a native speaker or by a professional language editor.

Use the cover letter as an opportunity to "sell your paper" to the editor. Include the following basic elements. (1) Your request: to submit the paper (mentioning its title) for publication in the journal (mentioning the journal's name). (2) A summary of the paper's significance (in 2-3 sentences): what relevant problem it addresses, its main finding, and an indication why this finding is important. (3) A statement of the paper's relevance to the journal's audience. A good argument would be that related work has previously been published in the same journal. Make sure that you cite this work, as this shows your knowledge of, and interest in, the journal. (4) Any information required by the journal, such as a statement that the material has not been submitted elsewhere or 
a statement about conflicts of interest. In addition to these basic elements, you can articulate specific issues related to your paper.

Once you have completed your submission, you should archive relevant information from the submission process such as the date of submission, the generated manuscript ID, and the PDF file together with the original "raw" files of your paper in one directory on your computer. Save your login details for the journal's submission system, the manuscript's ID, and the automatically generated e-mails you receive from the journal. Monitor the status of your submission regularly and contact the journal if the status is unclear or when a decision is taking too long (e.g., when you haven't heard from the journal within 2-3 months).

\section{Checklist for submitting a paper}

- Read your entire paper from beginning to end carefully one more time

- Check whether you have adhered $100 \%$ to the journal's specific author requirements

- Write a convincing cover letter including the following elements:

- the paper's title and your request to submit for publication,

- significance of the main findings for the field,

- relevance to the journal's audience,

- information required by the journal, and

- additional issues relating to the paper

- Follow the steps of the journal's online submission system

- Archive all relevant data from the submission

- Monitor the processing of your paper by the journal from time to time 